\title{
Enhancing Performance of Multibiometric System using Ant Colony Optimization based on Score Level Fusion
}

\author{
Sandip Kumar Singh Modak \\ Dept. of Computer science \&Eng \\ Birla Institute of Technology \\ Mesra, Ranchi, India
}

\author{
Vijay Kumar Jha \\ Dept.of Computer science \&Eng \\ Birla Institute of Technology \\ Mesra, Ranchi, India
}

\begin{abstract}
Unimodal biometric systems have several inherent problems such as intra-class variation, noisy-sensor data, spoofing attacks and non-universality. To overcome this limitation multibiometric is a good option where we can use two or more individual modalities. In this paper we propose a multibiometric system to enhance the performance and minimize the error rate using Ant colony optimization (ACO) based on score level fusion. This work extracts the feature from two different modalities namely face and iris (left/right). In this work we use ACO as an optimization technique to select the fusion parameter like weights for the different biometric matcher and fusion rule which is used further for score level fusion. The experimental results show that the multibiometric system using ACO based on sum rule is outperform than the other fusion rule like product, tanh and exponential sum.
\end{abstract}

\section{Keywords}

Unimodal, multibiometric, ant colony optimization, fingerprint, iris, particle swarm optimization, score level fusion.

\section{INTRODUCTION}

Biometric authentication is a system which is capable to identify a person based on the inherent physical or behavioral traits associated with that person [1].In recent year the application of biometric in authentication system increase gradually; one of the main reasons of its popularity is that biometric traits like fingerprint, face and iris feature of a person are same during the whole life time and it is not easily guess, forgotten and misplace. Biometric based system are more secure and accurate compare to the traditional system which is based on user personal identification number(PIN) and the user set PIN can be easily guess by the third party. Unimodal system which is based on single modalities has several inherent problems like noisy senses data, intra-class variation, spoofing attack and unaccepted error rate [2]. To overcome this limitation multibiometric is a good option where we can use more than one modality at a time to improve the performance and accuracy level of the systems. Fusion process plays an important role in multibiometric where the features of more than one modality are combined together [3].The whole fusion process can be classified as sensor level, feature level (combining feature from different biometrics), score level (combining the genuine and imposter score) and decision level (combing the decisions) [4]. Among all the fusion methods score level fusion very popular and simple, lots of research have been done in the area of score level fusion. Researcher preferred to use score level fusion due to ease of combining matching score. Further score level fusion technique can be categories as a) transformationbased score fusion like sum rule, weighted sum rule and product rule. b) Classifier based like support vector machine (svm) and c) density based fusion like likelihood ratio test [5].
Evolutionary approaches plays an important role in multibiometric, it generate a optimum solution among a large population and finally the optimum solution is achieve through the searching and updating the past history of the particle [6].ACO (ant colony optimization), PSO (particle swarm optimization) and GA (genetic algorithm) are all comes under these category.PSO based algorithm sometime face the problem of premature convergence and its somehow effect in to get the optimum solution [7]. Score normalization play an important role in fusion process of different biometric modalities. After the matching process the output score obtained by each individual modalities are may not be homogenous in nature and they need not be on the same numerical range. So normalization is a technique which convert the output score into a common range so that it is easy to get a final score and further it is used in a decision module to decide whether the claimed user is genuine or not. The obtained scored form face and hand-geometry are distance score, whereas in the case of fingerprint it is similarity score. The different types of normalization technique are Min-min, $\mathrm{z}$-score, double sigmoid and tan-h estimators, among them Min-max normalization technique is most popular and mostly used in fusion purpose in multibiometric [2].The remainder of this paper is organized as follow: in section 2 related works are presented; section 3 give details about ACO; section 4 give details about the proposed work; section 5 give details about experimental result and section 6 concludes the papers.

\section{RELATED WORK}

Lots of researches have been done in multibiometric based on score level fusion. In this section we only mention the related works of score level fusion which is based on evolutionary technique like Pso, Ga, Aco and other optimization technique. Dynamic selection of matching score was proposed by Tronci et al. [8] in which they show that the dynamic selection of matching score can provide a better performance than a unimodal system. Veeramachaneni et al. [9] was proposed an adaptive multimodal biometric management algorithm which is based on the combination of PSO and Bayesian decision fusion. In this paper the system has $\mathrm{N}$ biometric sensors to collect the data, a particle swarm optimizer (PSO), Bayesian decision fusion processor and a mission manager. Bayesian decision fusion processor is used to fuse the decisions from different biometric sensors and the optimal fusion rule from PSO into a final decision.Srinivas et al. [10] proposed a system for enhancing the performance of correlated biometric classifiers, in which weighted sum rule with the combination of PSO is used in score level fusion. The weights of individual classifier are calculated using the concept of PSO. Raghavendra et al. [11] proposed a PSO based fusion of near infrared and visible image for improved face verification, in the first scheme PSO is used to calculate the optimum weight of a weighted linear combination of the coefficients and in the $2^{\text {nd }}$ scheme PSO is used to select the optimal fused feature of near infrared and visible image. L. Mezai and F.Hachouf [6] 
proposed a fusion of face and voice using PSO and belief function at score level fusion, in which the belief assignment is generated from the score of each modality using Denoeux and Appriou models.PSO is used to estimates the confidence factor, the fusion of weighted belief assignment is carried out using Dempster-Shafer(DS) and finally make decision making whether the claim user is genuine or not. Kumar et al. [12] proposed an adaptive combination of multiple biometrics classifier based on evolutionary approach in which the hybrid PSO model is used to obtain the different fusion parameter and optimal fusion strategy. The authors use different possible methods like simple sum, weighted sum, product and exponential sum as a score level fusion strategy. They have uses different combination of modalities like combination of iris and palmprint, face and speech and fingerprint and hand geometry to carry out their experiment. Amioy Kumar and Ajay Kumar [13] proposed a multimodal biometric fusion using ant colony optimization (ACO) in which they shows their experiment results using publicly available database like IIITD database of palmprint and iris; database from speech and face, and the NIST database of fingerprint and face images. Kumar et al. [14] proposed an ant colony optimization (ACO) based fuzzy binary decision tree for bimodal hand knuckle verification system, in which they uses ACO to choose optimal fusion parameter for each level of security.FBDT (fuzzy binary decision tree) are used for decision making purpose and classify the classes as genuine or imposter using matching score obtained from knuckle database. The application of GA (genetic algorithm) for selection of different fusion parameter at score level fusion was proposed by Romain Giot et al. [15] in which they define a fitness function based on a fast Error Equal Rate computing method. They have tested three different kinds of score fusion methods whose parameter are automatically set by genetic algorithm, the score fusion functions have been validate on three different multibiometric database on which two are real and one is chimerical.Cherifi Dalila and Hafnaoui Imane [16] proposed a multibiometric system using combination of GA and PSO based on score level fusion, in which PSO and GA is applied to find the optimum weights associated to the modalities being fused. They conclude that the performance of hybrid GA with PSO, GA and PSO at score level is differing significantly in terms of time consumption.Aniesha et al. [17] proposed a Genetic and Evolutionary computation (GEC)-based multibiometric fusion scheme to optimize the weights assigned to the different biometric modalities of a multibiometric system for score level fusion, the modalities tested were face and periocular biometrics. They conclude that the after using the concept of GEC at score level, it can help to improve the recognition accuracy of the system.

\section{ANT COLONY OPTIMIZATION}

Ant colony optimization [18] was first introduce by Marco Dorigo in the year 1992 is a one kind of probabilistic technique used to find the optimal solution of a problem based on the behavior of the ant. This algorithm is a member of the ant colony algorithm family, in swarm intelligence methods, and it constitute some metaheuristic optimization. Initially each ant has an equal amount of pheromone (a chemical substance produced and released into the environment by an ant) level, using that they direct each other to the resource and similarly record their position to obtain the better solution in next iteration. Two important factors in ACO are Q (pheromone constant) and $\rho$ (evaporation factor) whose value is less than 1.Initially each ant select its possible solution randomly from the set of available possible values.
For each ant, the objective function $\mathrm{G}$ (shown in Eqn. 2) is calculated and pheromone level of all the solution are updated using the initial pheromone level, computed function E, pheromone constant $\mathrm{Q}$ and evaporation factor $\rho$ as follow:

$\tau_{i}(t+1)=\rho * \tau_{i}(t)+\frac{Q}{E} \quad$ If $\mathrm{i}^{\text {th }}$ solution is selected in $\mathrm{i}^{\text {th }}$ iteration

$=\rho * \tau_{i}(t)$ If $\mathrm{i}^{\text {th }}$ solution is not selected in $\mathrm{i}^{\text {th }}$

iteration

Where $\mathrm{Q}=$ pheromone constant, $\mathrm{E}=$ Error calculated, $\rho$ =evaporation constant and $\tau_{i}(t)=$ pheromone level at $\mathrm{t}^{\text {th }}$ iteration. The objective function is given by Eqn. 2 .

$G=C F A * F A R(\alpha)+C F R * F R R(\alpha) \quad \ldots \ldots$

Where $C F A+C F R=2$

\subsection{Bimodal fusion strategy}

Researcher preferred to use score level and decision level as a fusion strategy in multibiometric system employed for the fusion of two or more biometric modalities. In this work we used score level fusion for fingerprint and iris, and finally ACO is used for optimal selection of fusion parameter like different weight for output matcher by different modalities and fusion rule. FAR (false acceptance rate) and FRR (false rejection rate) are two key parameter for measure the performance of any biometric system. FAR is the rate at which imposter user are accepted as genuine users while FRR is the rate at which genuine users are rejected considering imposter ones. Both FAR and FRR are complement to each other and for achieve high level of security it is mandatory to maintain the value of FAR and FRR as low as possible. But it is very difficult to set both these value low at the same time.EER (equal error rate) is another key parameter to measure the performance of the multibiometric system which is a value at which both FAR and FRR are equal. The cost of false acceptance (CFA) and cost of false rejection (CFR) are two parameters through which we can measure the security level of the system. Both of these cost belong to $[0,2]$ in the step of 0.1. The four popular score level fusion rule are sum rule, product rule, exponential sum and Tan-hyperbolic (Tanh) which are as follows:

$$
\begin{aligned}
& \text { Sum }=\sum_{j=1}^{2} s_{j} w_{j} \\
& \text { Product }=\prod_{j=1}^{2} s_{j}{ }^{w_{j}}
\end{aligned}
$$$$
\text { Exponential Sum }=\sum_{j=1}^{2} \exp \left(s_{j}\right) w_{j} \ldots \ldots \ldots
$$

Tanh Sum $=\sum_{j=1}^{2} \tanh \left(s_{j}\right) w_{j}$

Where $s_{j}$ be the matching score obtained by the $\mathrm{j}^{\text {th }}$ modality and $w_{j}$ be the corresponding weight assign for $\mathrm{j}=1,2$.

\subsection{ACO for proposed bimodal}

Each ant in the search space is represented by $x_{m d}=$ $\left(x_{m 1}, x_{m 2}, \ldots \ldots, x_{m D}\right)$, where the first subscript $\mathrm{m}$ denotes a mth solution and $\mathrm{D}$ denote the dimension. As we use score level fusion for two modalities so each ant can be represented by " $\mathrm{N}+1$ " dimensions where $\mathrm{N}$ denotes the total number of modality which is 2 in this case and the last dimension is for fusion rule chosen from Eqs (3)- (6). Each ant can be represented by $x_{m d}=\left\{w_{1}, w_{2}, F\right\}$, where first two parameters are the weights assigned and last parameter is the fusion rule. Initially each ant is assign with the equal amount of pheromone level. To obtain the fused matched 
score the individual weights assign by the ACO is applied to the corresponding matching score and fusion is performed with the chosen fusion rule like weighed sum,product,tanh and exponential sum. For each ant the value of G (Eqn. 2) is calculated and that fusion parameter is selected as optimum which minimizes the value of G.the update on the pheromone level at $(t+1)$ th iteration where $i$ denote the ith ant is given in Eqn. 1. The main problem with the ACO technique is the saturation of pheromone level for small value of $\rho$ (approx. 0) and forcing the ant stuck on a particular path. To overcome this problem if we set the value of $\rho$ to approx. 1 then every path will have the same probability and there is no scope of optimization. So better solution of this problem is to initially set the value of $\rho$ near to 1 and gradually decrease the value in steps of 0.005 .

\section{PROPOSED WORK}

In this paper we propose a multibiometric system using ACO based on score level fusion to enhance the overall system performance. This work extract feature from two biometric modalities namely faces and iris (left/right). In the enrollment phase the extracted features from two different biometric modalities face and iris (left/right) are stored in the respective database which is shown in Fig 1. During the authentication phase the extracted face and iris (left/right) features of claimed identity is compared with the existing stored template of face and iris, which in result produce three match score namely $w_{1}, w_{2}$ and $w_{3}$. In this work we use ACO as an optimization technique to select the fusion parameter like weights for the different biometric matcher $\left(w_{1}, w_{2}, w_{3}\right)$ and fusion rule which is used further for score level fusion. And finally the claimed identity is considered genuine if the resultant score value is above the system threshold value, otherwise the claimed identity is considered as imposter shown in Fig 2.

\subsection{Facial feature extraction}

The various phases involve in feature extraction of face are pre-processing, Image-enhancement and feature extraction. In the face recognition system the first phase is pre-processing in which face image are detect using the Active Appearance Modeling (AAM toolbox) [19] used to locate the center position of the left and right iris of eyes. This toolbox is helped to measure the angle of head roll that may be occurred during the acquisition of the face image. By using the center position and measured angle both eyes are aligned in the face image. After pre-processing the next step is image enhancement used to enhance the image quality. For this purpose we use Histogram Equalization (HE) and meanvariance normalization (MVN) [20] which is apply on resized image of size $60 \times 60$ to reduce the effect of illumination, finally Log-Gabor transform technique is used for facial feature extraction to transform the image of size $40 \times 80$.Finally, match scores are produced using the Manhattan distance measurement.

\subsection{Iris feature extraction}

The various phases of iris feature extraction are as follow

\subsubsection{Segmentation}

The main goal of segmentation is to isolate the require iris region from the whole eye image by separating the part of an image between the inner boundary and outer boundary. Two well known iris segmentation method are attributed to Daugman and Wildes. Daugman developed integrodifferential operator to find circular pupils and limbus boundaries. It can be interpreted as a circular edge detector, which searches in a smoothed image by Gaussian filter, the parameter of a circular boundary along which the integra derivative is maximal [21].Wilds proposed a two stage iris segmentation method gradient based intensity image, and next the inner and outer boundaries are detected using Hough transform[22].

\subsubsection{Normalization}

The extracted iris region is then normalized into a fixed rectangular box. The most common normalization method proposed by Daugman. According to Daugman's rubber sheet model, the remapping of the iris image $\mathrm{I}(\mathrm{x}(\mathrm{r}, \Theta), \mathrm{y}(\mathrm{r}$, $\Theta)) \rightarrow \mathrm{I}(\mathrm{r}, \Theta)$ from Cartesian coordinate to doubly dimensionless pseudo polar coordinate system can be represented as

$x(r, \varnothing)=(1-r) x_{p}(\varnothing)+r x_{s}(\varnothing)$

$y(r, \varnothing)=(1-r) y_{p}(\varnothing)+r y_{s}(\varnothing)$

Where $\left(x_{p}(\varnothing), y_{p}(\varnothing)\right)$ and $\left(x_{s}(\varnothing), y_{s}(\varnothing)\right)$ are respectively the discrete co-ordinate nearest to the papillary boundary and $\mathrm{r}$ is the normalized radius in the interval $[0,1]$.

\subsubsection{Feature extraction}

In this step the unique pattern of iris is extracted using LogGabor transform with the same strategy as in face recognition. And the final size of the Log-Gabor transform image is set to $40 \times 80$. Finally, match scores are produced using the Manhattan distance measurement.

\section{EXPERIMENTAL RESULTS}

In this paper fusion of face and iris (left/right) at score level fusion is presented. We considered four bimodal systems these are a) face and left iris b) face and right iris c) left iris and right iris d) face, left iris and right iris.

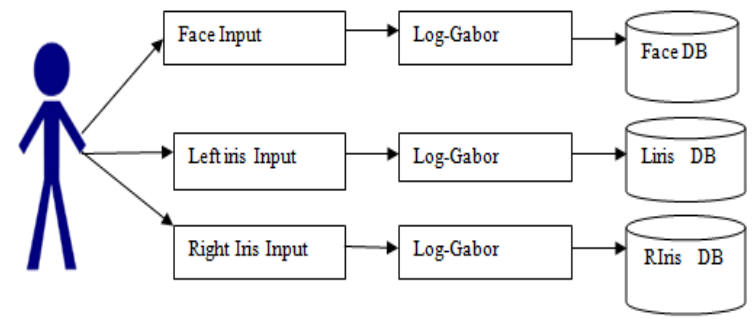

Fig 1: Enrollment Phase

Fusion parameter in Aco based learning is mostly depends on the cost factor CFA; so the ACO algorithm runs for each value of CFA.The fusion parameter are computed for each value of CFA and initially the value of $\rho$ is set to 1 which is decrease in step of 0.005 to get the global optimal value. Experiment are carried out in the range of $[0.005,0.01]$ and it is found that $\mathrm{Q}=0.01$ is acceptable. In this experiment we select total 15 numbers of ants which is found enough for the convergence of the algorithm. The number of iteration for ACO algorithm is taken as 50 and found sufficient for the convergence of the solution. The whole experiment are carried out using on a publicly available database called CASIA-IRIS-DISTANCE [23] which is a combination of both dual-eye iris and facial feature of 2567 images of 142 subjects. The average size of extracted iris is $170 \times 150$, and the average number of pixels between irises is 760.It was implemented in MATLAB R2016a.we consider randomly 90 subjects and each subject possess 10 sample, on which 5 sample for references and 5 for testing which is performed 10 times without any overlapping. In each trial we got 
$(90 \times 5=450)$ genuine score by compare the image of same person and $40,050(90 \times 89 \times 5)$ imposter score by compare an image of a person with an image of another person. The performance of the system is measured by FAR (False acceptance rate), GAR (Genuine acceptance rate), and TER (Total error rate).It is represented by the ROC (Receiver Operating Characteristic) curve which plots FAR probability versus GAR probability for different value of the decision threshold. Table 1-4 shows the values of FAR and FRR correspond to the value of decision threshold for fusion of (face and left iris), (face and right iris), (left iris and right iris) and (face, left iris and right iris).

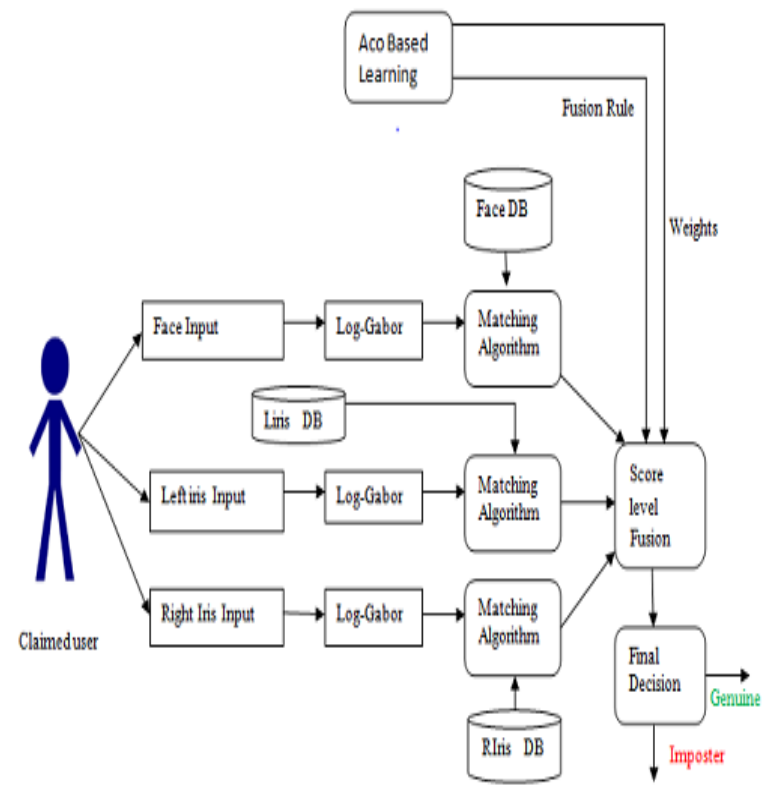

Fig 2: Authentication Phase

Table 1: FAR and FRR for fusion of face and left iris

\begin{tabular}{|c|c|c|}
\hline Threshold & FAR\% & FRR\% \\
\hline 0.8 & 21.2 & 2.0 \\
\hline 0.9 & 17.4 & 2.6 \\
\hline 1.0 & 14.5 & 3.1 \\
\hline 1.1 & 10.2 & 5.4 \\
\hline 1.2 & 8.9 & 7.8 \\
\hline 1.3 & 6.2 & 9.3 \\
\hline 1.4 & 2.9 & 11.6 \\
\hline 1.5 & 0.9 & 15.1 \\
\hline 1.6 & 0.5 & 17.4 \\
\hline 1.7 & 0.2 & 19.2 \\
\hline 1.8 & 0.15 & 21.9 \\
\hline
\end{tabular}

For each bimodal system the minimum overall error rate computed corresponding to each CFA is shown in Fig. 3a-d, respectively.
Table 2: FAR and FRR for fusion of face and right iris

\begin{tabular}{|c|c|c|}
\hline Threshold & FAR $\%$ & FRR $\%$ \\
\hline 0.8 & 22.4 & 2.0 \\
\hline 0.9 & 18.4 & 2.4 \\
\hline 1.0 & 15.5 & 3.5 \\
\hline 1.1 & 9.2 & 5.6 \\
\hline 1.2 & 7.9 & 7.7 \\
\hline 1.3 & 5.2 & 9.2 \\
\hline 1.4 & 2.3 & 11.1 \\
\hline 1.5 & 1.9 & 15.4 \\
\hline 1.6 & 0.9 & 17.2 \\
\hline 1.7 & 0.5 & 19.4 \\
\hline 1.8 & 0.2 & 21.8 \\
\hline
\end{tabular}

Table 3: FAR and FRR for fusion of left and right iris

\begin{tabular}{|c|c|c|}
\hline Threshold & FAR\% & FRR\% \\
\hline 0.40 & 12.4 & 0.7 \\
\hline 0.41 & 10.5 & 1.6 \\
\hline 0.42 & 9.6 & 2.8 \\
\hline 0.43 & 7.4 & 3.4 \\
\hline 0.44 & 5.9 & 5.5 \\
\hline 0.45 & 3.2 & 6.3 \\
\hline 0.46 & 2.4 & 8.4 \\
\hline 0.47 & 1.2 & 10.2 \\
\hline 0.48 & 0.8 & 13.4 \\
\hline 0.49 & 0.5 & 14.9 \\
\hline 0.5 & 0.15 & 15.2 \\
\hline
\end{tabular}

Table 4: FAR and FRR for fusion of face, left and right iris

\begin{tabular}{|c|c|c|}
\hline Threshold & FAR\% & FRR\% \\
\hline 0.40 & 12.2 & 0.6 \\
\hline 0.41 & 9.5 & 1.04 \\
\hline 0.42 & 9.1 & 2.6 \\
\hline 0.43 & 7.4 & 3.14 \\
\hline 0.44 & 5.8 & 5.35 \\
\hline 0.45 & 3.3 & 5.99 \\
\hline 0.46 & 2.3 & 7.89 \\
\hline 0.47 & 1.1 & 9.2 \\
\hline 0.48 & 0.86 & 10.4 \\
\hline 0.49 & 0.5 & 11.9 \\
\hline 0.5 & 0.18 & 13.2 \\
\hline
\end{tabular}

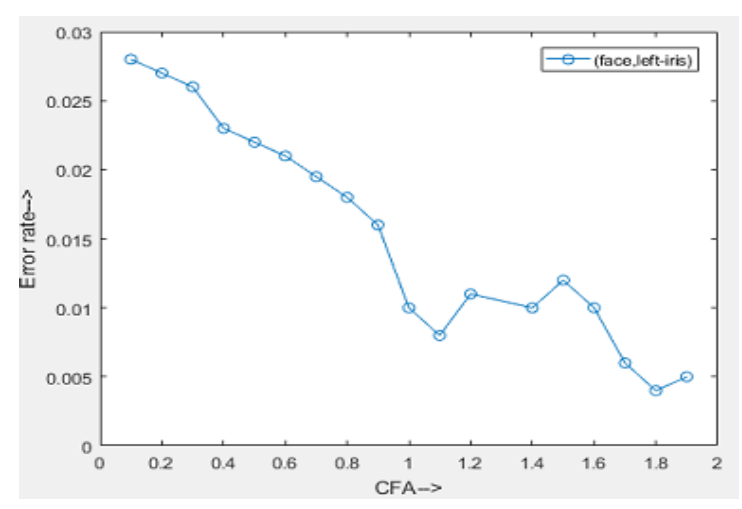

Fig.3 (a): CFA vs. Error rate for face and left iris 


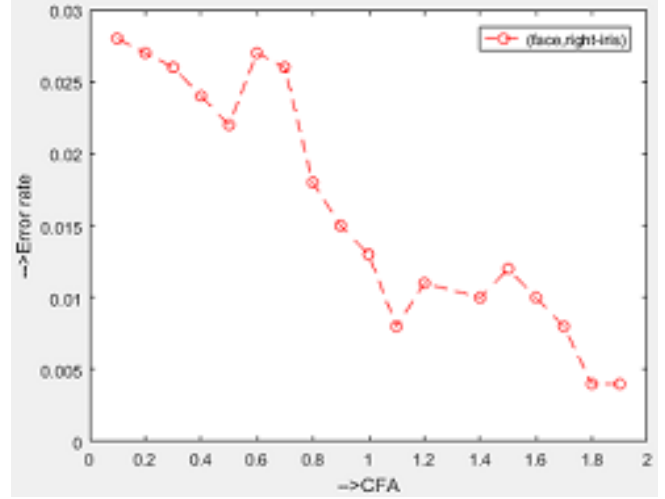

Fig.3 (b): CFA vs. Error rate for face and right iris

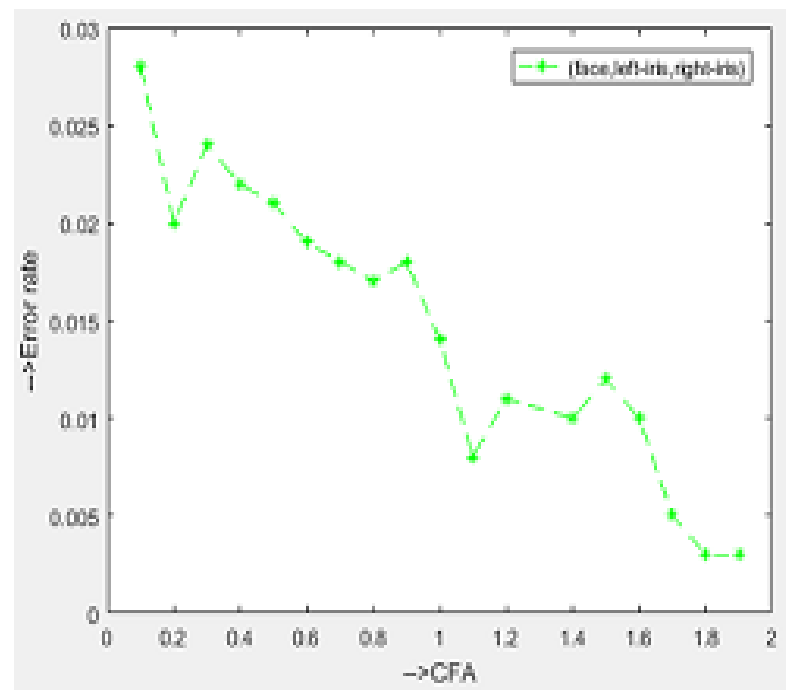

Fig.3 (d): CFA vs. Error rate for face, left and right iris

\section{CONCLUSIONS}

In this paper we propose a multibiometric system using ant colony optimization (ACO) as an optimization technique based on score level fusion to improve the performance and reduce the error rate of the proposed system. The role of ACO is to select the most optimal value of fusion parameter like fusion rule and the weights assign to the different biometric matcher. The security level of the system is totally depends on two parameter like FAR (false acceptance rate) and FRR (false rejection rate). The ACO is selected to choose optimal fusion parameter corresponding to each CFA, which denotes the different security level in the bimodal system. For obtained the high security level in the bimodal system the cost of false acceptance rate(FAR) can be set higher than those of false rejection rate(FRR) because imposter acceptance should be as less as possible. In order to obtain the low security we choose the cost of FRR much higher than the FAR because in this case the genuine rejection is required to be maintain as less as possible. In bimodal system CFA (cost of false acceptance) and CFR (cost of false rejection) are used to measure the security level of the system. We considered four bimodal systems these are a) face and left iris b) face and right iris c) left iris and right iris d) face, left iris and right iris. The experimental result shows that among the several fusion rules (sum, exponential sum, tanh and product), the sum rule is one

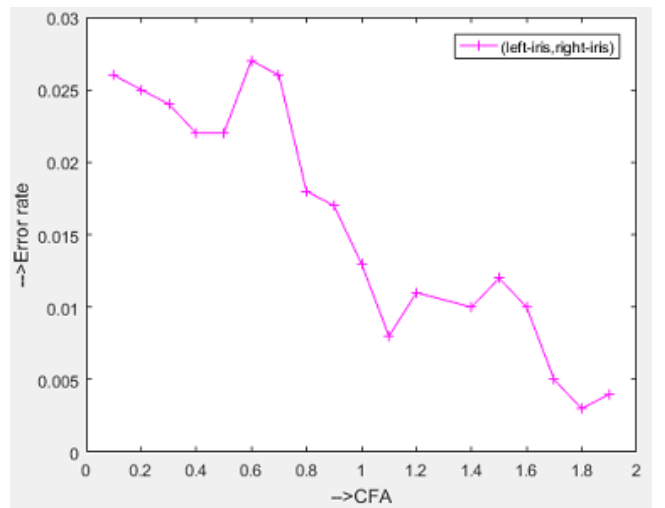

Fig.3 (c): CFA vs. Error rate for left and right iris

Table 5: ACO based Error rate for different fusion rule

\begin{tabular}{|c|c|c|c|c|c|c|c|c|}
\hline \multirow[t]{2}{*}{ CFA } & \multicolumn{2}{|c|}{ Easetleftitis } & \multicolumn{2}{|c|}{ Facet rightirs } & \multicolumn{2}{|c|}{ left iris + right iris } & \multicolumn{2}{|c|}{ face-left iris + right iris } \\
\hline & Errors & Fusiom rule & Errors & Fusion rals: & Errors & Fusion rule & Errors & Fusion rule \\
\hline 0.1 & 0.025 & Sum & 0.024 & Sum & 0.023 & Sum & 0.025 & Sum \\
\hline 0.2 & 0.023 & Product & 0.021 & Sum & 0.022 & Sum & 0.022 & Product \\
\hline 0.3 & 0.021 & Exp. Sum & 0.020 & Product & 0.019 & Sum & 0.020 & Tanh \\
\hline 0.4 & 0.020 & Product & 0.022 & Exp Sum & 0.021 & Product & 0.021 & $S u m$ \\
\hline 0.5 & 0.018 & Tanh & 0.017 & Sum & 0.016 & Product & 0.017 & Sum \\
\hline 0.6 & 0.015 & Product & 0.014 & Product & 0.013 & Sum & 0.014 & Sum \\
\hline 0.7 & 0.013 & Sum & 0.013 & Sum & 0.012 & Exp. Sum & 0.011 & $\operatorname{Tamh}$ \\
\hline 0.8 & 0.011 & Sum & 0.012 & Product & 0.015 & Product & 0.013 & Sum \\
\hline 0.9 & 0.001 & Sum & 0.002 & Product & 0.002 & Sum & 0.003 & Sum \\
\hline 1.0 & 0.008 & Product & 0.007 & Sum & 0.006 & Sum & 0.005 & Sum \\
\hline 1.1 & 0.004 & Sum & 0.003 & Produer & 0.004 & Sum & 0.002 & Sum \\
\hline 1.2 & 0.003 & Sum & 0.001 & Product & 0.001 & Sum & 0.0015 & Sum \\
\hline
\end{tabular}

of the best fusion rule with minimum error rate and its accuracy level is outperformer than the other fusion rule.

\section{REFERENCES}

[1] A.K.Jain, A.Ross, and S.Prabhakar. "An Introduction to biometric recognition", IEEE Transaction on Circuits and Systems for Video Technology. Special Issue on Image and Video-Based Biometrics, vol.14 (1):pp.4-20, 2004.

[2] Anil Jain,Karthik Nandakumar, Arun Ross," Score Normalization in Multimodal Biometric System", journal of Pattern Recognition, Vol.38,pp.2270-2285,2005.

[3] He, Mingxing, et al. "Performance evaluation of score level fusion in multimodal biometric systems", Pattern Recognition, vol. 43.5, pp: 1789-1800, 2010.

[4] Hanmandlu, Madasu, et al. "Score level fusion of multimodal biometrics using triangular norms", Pattern recognition letters, vol. 32.14, pp: 1843-1850, 2011.

[5] Nandakumar, Karthik, et al. "Likelihood ratio-based biometric score fusion", IEEE transactions on pattern analysis and machine intelligence, vol.30.2, pp: 342-347, 2008. 
[6] Mezai, Lamia, and Fella Hachouf. "Score-level fusion of face and voice using particle swarm optimization and belief functions", IEEE Transactions on Human-Machine Systems, vol. 45.6, pp: 761-772, 2015.

[7] Kumar, Amioy, et al. "Decision level biometric fusion using Ant Colony Optimization", Image Processing (ICIP), 2010 17th IEEE International Conference on. IEEE, 2010.

[8] Tronci, Roberto, Giorgio Giacinto, and Fabio Roli. "Dynamic score selection for fusion of multiple biometric matchers", Image Analysis and Processing, 2007. ICIAP 2007. 14th International Conference on. IEEE, 2007.

[9] Veeramachaneni, Kalyan, Lisa Ann Osadciw, and Pramod K. Varshney. "An adaptive multimodal biometric management algorithm", IEEE Transactions on Systems, Man, and Cybernetics, Part C (Applications and Reviews), vol.35.3, pp: 344-356,2005.

[10] Srinivas, Nisha, Kalyan Veeramachaneni, and Lisa Ann Osadciw. "Fusing correlated data from multiple classifiers for improved biometric verification", Information Fusion, 2009. FUSION'09. 12th International Conference on. IEEE, 2009.

[11] Raghavendra, Ramachandra, et al. "Particle swarm optimization based fusion of near infrared and visible images for improved face verification", Pattern Recognition, vol. 44.2, pp: 401-411, 2011.

[12] Kumar, Ajay, Vivek Kanhangad, and David Zhang. "A new framework for adaptive multimodal biometrics management", IEEE transactions on Information Forensics and Security, vol. 5.1, pp: 92-102, 2010.

[13] Kumar, Amioy, and Ajay Kumar. "Adaptive management of multimodal biometrics fusion using ant colony optimization", Information Fusion, vol. 32, pp: 49-63, 2016.

[14] Kumar, Amioy, Madasu Hanmandlu, and H. M. Gupta. "Ant colony optimization based fuzzy binary decision tree for bimodal hand knuckle verification system", Expert Systems with Applications, vol. 40.2, pp: 439449, 2013.

[15] Giot, Romain, Mohamad El-Abed, and Christophe Rosenberger. "Fast learning for multibiometric systems using genetic algorithms", High Performance Computing and Simulation (HPCS), 2010 International Conference on. IEEE, 2010.

[16] Cherifi, Dalila, Imane Hafnaoui, and Amine Nait Ali. "Multimodal score-level fusion using hybrid ga-pso for multibiometric system." (2015).

[17] Alford, Aniesha, et al. "GEC-based multi-biometric fusion", Evolutionary Computation (CEC), 2011 IEEE Congress on. IEEE, 2011.

[18] Dorigo, Marco, and Luca Maria Gambardella. "Ant colony system: a cooperative learning approach to the traveling salesman problem", IEEE Transactions on evolutionary computation, vol. 1.1, pp: 53-66, 1997.

[19] Huang, Chen, Xiaoqing Ding, and Chi Fang. "Pose robust face tracking by combining view-based AAMs and temporal filters", Computer Vision and Image Understanding, vol.116.7, pp: 777-792, 2012.

[20] Pujol, Pere, Dusan Macho, and Climent Nadeu. "On realtime mean-and-variance normalization of speech recognition features", Acoustics, Speech and Signal Processing, 2006. ICASSP 2006 Proceedings. 2006 IEEE International Conference on. Vol. 1. IEEE, 2006.

[21] Daugman, John G. "High confidence visual recognition of persons by a test of statistical independence", IEEE transactions on pattern analysis and machine intelligence, vol.15.11, pp: 1148-1161, 1993.

[22] Wildes, Richard P. "Iris recognition: an emerging biometric technology", Proceedings of the IEEE, vol. 85.9, pp: 1348-1363, 1997.

[23] Biometric Ideal Test. Available online: http://biometrics.idealtest.org/dbDeatailForUser.do?id=4 (assessed on 30 August 2013). 